\title{
Demographic and Clinical Characteristics of Patients with Sustained and Switching Treatments Using Biological and Targeted Synthetic Disease-Modifying Antirheumatic Drugs: A Multicenter, Observational Cross-Sectional Study for Rheumatoid Arthritis
}

\author{
Sebnem Ataman · Ismihan Sunar (D) - Hatice Bodur • Meltem Alkan Melikoglu • Hasan Fatih Cay • \\ Erhan Capkin · Ozgur Akgul • Remzi Cevik · Feride Gogus • Ayhan Kamanli · Fatma Gul Yurdakul • \\ Gulcan Gurer · Ilker Yagci · Aylin Rezvani · Mehmet Tuncay Duruoz
}

Received: August 8, 2021 / Accepted: November 16, 2021 / Published online: December 1, 2021

(c) The Author(s) 2021

\section{ABSTRACT}

Introduction: Rheumatoid arthritis is a chronic inflammatory disease with different disease activity grades. Several registries have been designed to determine the appropriate regimens of disease-modifying antirheumatic drugs to

\section{S. Ataman}

Division of Rheumatology, Department of Physical Medicine and Rehabilitation, Faculty of Medicine, Ankara University, Ankara, Turkey

I. Sunar $(\bowtie)$

Rheumatology Clinic, Aydın State Hospital, Aydın, Turkey

e-mail: dr.ismihan@gmail.com

H. Bodur · F. G. Yurdakul

Department of Physical Medicine and

Rehabilitation, Ankara City Hospital, Ankara,

Turkey

\section{A. Melikoglu}

Division of Rheumatology, Department of Physical Medicine and Rehabilitation, Ataturk University

School of Medicine, Erzurum, Turkey

\section{H. F. Cay}

Department of Rheumatology, Antalya Training and Research Hospital, University of Health Sciences, Antalya, Turkey

\section{E. Capkin}

Department of Physical Medicine and Rehabilitation, Karadeniz Technical University School of Medicine, Trabzon, Turkey obtain sustained clinical remission. We examined epidemiological and clinical characteristics of rheumatoid arthritis patients using a clinical registry database (BioSTaR) and analyzed the differences in patients with sustained and switched therapies.

Methods: A multicenter, observational crosssectional study for rheumatoid arthritis was

\section{O. Akgul}

Division of Rheumatology, Department of Physical Medicine and Rehabilitation, Manisa Celal Bayar University School of Medicine, Manisa, Turkey

\section{R. Cevik}

Department of Physical Medicine and Rehabilitation, Dicle University School of Medicine, Diyarbakır, Turkey

\section{F. Gogus}

Division of Rheumatology, Department of Physical Medicine and Rehabilitation, Gazi University School of Medicine, Ankara, Turkey

\section{A. Kamanli}

Division of Rheumatology, Department of Physical Medicine and Rehabilitation, Sakarya University School of Medicine, Sakarya, Turkey

\section{G. Gurer}

Division of Rheumatology, Department of Physical Medicine and Rehabilitation, Adnan Menderes University School of Medicine, Aydın, Turkey

\section{Yagci}

Department of Physical Medicine and Rehabilitation, Marmara University School of Medicine, Istanbul, Turkey 
performed between February 2019 and September 2020 using the BioStaR-RA registry.Demographic and clinical characteristics were prospectively recorded into a specifically designed electronic database. The patients were divided into three groups due to the heterogeneity of the study cohort. Patients were grouped as Group I (Initial; within the first 6 months of treatment with biological/targeted synthetic drugs), Group ST (Sustained Treatment; any first drug lasting for at least 6 months without any change), and Group S (Switch; any switching to another drug). Comparative analysis was performed between sustained treatment (Group ST) and drug switching (Group S) groups.

Results: The study included a total of 565 patients. The mean age was $53.7 \pm 12.8$ years, and the majority were female $(80.4 \%)$. There were 104, 267, and 194 patients in Groups I, ST, and S, respectively. Erosive arthritis and hematological extra-articular involvement were more frequently detected in Group S than Group ST $(p=0.009$ and $p=0.001)$. The patients in Group $S$ had significantly higher disease activity scores (DAS28-CRP, CDAI, and SDAI) $(p=0.025$, $p=0.010$, and $p=0.003)$. There were significantly more patients with moderate disease activity in Group $S(p<0.05)$.

Conclusions: The groups with sustained treatment and switching included patients with different disease activity status, although higher disease activity was determined in switchers. Overall, moderate disease activity and remission were the most common disease activity levels. Lower disease activity scores, lower hematologic manifestations, better functional status, and lesser radiographic damage are associated with sustained treatment.

\footnotetext{
A. Rezvani

Department of Physical Medicine and

Rehabilitation, Medipol University School of

Medicine, Istanbul, Turkey

M. T. Duruoz

Division of Rheumatology, Department of Physical Medicine and Rehabilitation, Marmara University

School of Medicine, Istanbul, Turkey
}

Keywords: Rheumatoid arthritis; Diseasemodifying antirheumatic drugs; Registries; Treatment switching

\section{Key Summary Points}

The biological and targeted synthetic disease-modifying antirheumatic drugs (DMARDs) have led to remarkable improvements in the management of rheumatoid arthritis (RA). The current study aimed to present real-life results of our biologic/targeted synthetic therapy registry of the BioSTaR.

We have analyzed the clinical differences in patients with sustained and switched therapies. We have observed that the most prevalent disease activity levels were low and moderate disease activity both in the sustained and switch therapy groups. While low disease activity is indicated as an acceptable disease activity status, patients with moderate disease activity are not a minority despite the advanced management strategies. These patients do not have the chance to alter their therapies due to local reimbursement conditions. Patients with sustained treatment had lower disease activity scores and hematologic manifestations, and better functional and radiographic status.

\section{INTRODUCTION}

Rheumatoid arthritis (RA) is one of the chronic inflammatory diseases that cause erosive arthritis and consequently functional loss if it is not treated with early effective treatment modalities [1, 2]. Remission rates usually depend on several factors belonging to the patients and the medications used for RA. The use of biological and targeted synthetic diseasemodifying antirheumatic drugs (bDMARDs, tsDMARDs) has improved the clinical course of the disease with better clinical outcomes [3]. 
Sustained clinical remission or at least low disease activity (LDA) has become the standard of care for patients with RA based on The European League Against Rheumatism (EULAR) statements [1, 4-6]. Sustained clinical remission is directly related to the achievement of functional remission. Switching between different bDMARDs is a frequent reality to achieve such therapeutic goals [7]. However, the association between sustained clinical remission and the status of drug switching has remained largely unknown $[6,8]$. An opposite correlation was reported between the proportion of patients with remission or low disease activity and the number of switches [9]. Thus, a personalized approach may be required to achieve a better clinical outcome.

Several randomized clinical trials and crosssectional epidemiological studies have investigated the predictors of response to biological therapies in achieving remission. However, it has been thought that strict inclusion criteria and relatively small sample sizes in these trials prevent the generalizability of the outcomes to the clinical practice [3]. We think that investigating RA patients on bDMARDs beyond the prespecified time-points after treatment may help physicians assess the disease's long-term outcome associated with the different disease categories [10].

Registries can be used to take real-world data and support the implementation of treat-totarget strategies about the most common forms of chronic inflammatory conditions $[3,5]$. It is believed that the outcomes of the registries are more applicable to clinical practice [11]. The BioSTAR-RA (Biologic, conventional Synthetic and Targeted synthetic Antirheumatic drugs Registry for Rheumatoid Arthritis) is a registry project developed by the Turkish League Against Rheumatism. This system collects the course of RA in Turkey and monitors the treatment applications in RA patients. It is known that physicians' treatment decisions regarding advanced therapies of RA patients are commonly affected by the patient's clinical factors, whereas healthcare policy is also regarded [12]. In this context, the analyses from this cohort will aid in identifying the treatment sequences and reasons for switching in RA patients receiving bDMARD/tsDMARD in a real-world setting.

This study aimed to present the epidemiological and clinical characteristics of the patients from the BioSTAR-RA registry and compare the groups of sustained and switch therapies of bDMARDs or tsDMARDs in terms of demographic and clinical factors. We also analyzed the characteristics of the patients within the first 6 months of these DMARDs treatments.

\section{METHODS}

\section{Study}

A multicenter, observational cross-sectional study was planned to evaluate the patients with RA between February 2019 and September 2020 using the BioStaR-RA registry. It is a registry that intends to involve prospective data (visits of every 6 months) of RA patients with advanced treatments, including bDMARDs and tsDMARDs. In this study, we analyzed only the first visits of each patient. All data in this registry were anonymized for the protection of the confidentiality and privacy of the patients. There were 13 centers from Turkey attending this study. The local ethical committee approved the study (Ankara Numune Training and Research Hospital, Number: E-182413; Turkish Medicine and Medical Devices Agency, 66175679-514.99-E182413). This study was carried out in compliance with the Declaration of Helsinki. Written informed consent was taken from the participants (Fig. 1).

\section{Patients}

The patients who fulfilled the diagnostic criteria of RA by the 2010 American College of Rheumatology (ACR)/European League Against Rheumatism (EULAR) classification criteria were recorded into a predefined database [13]. The patients whose age was between 18 and 70 years were included. Patients with recent malignancy history ( $<5$ years), chronic demyelinating disease, active tuberculosis, severe heart failure, and ones not approving to participate in the 


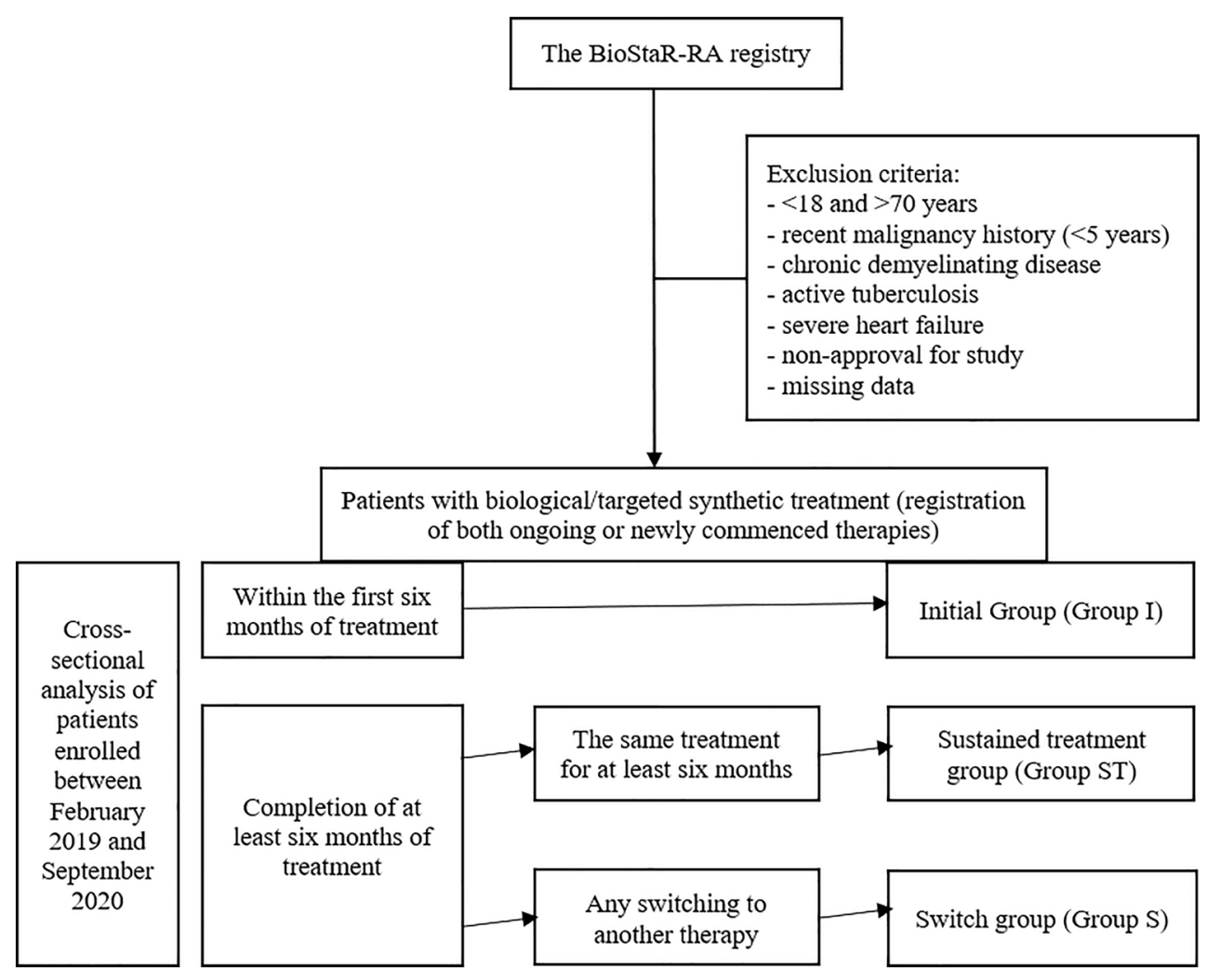

Fig. 1 Flowchart of the study

study were excluded from the BioSTAR Registry, and patients with missing data were not included in these analyses.

\section{Variables}

We used a specifically designed electronic database for the entrance of medical data collected directly from the patients. Other medical information was obtained from each institution's hospital information system. Demographic data, including age, sex, weight, and height, the district area of Turkey in which the patients lived, marital and educational status, and history of smoking and alcohol habits were recorded at the admission to the registry. Body mass index (BMI) was calculated as total body weight $(\mathrm{kg})$ divided by the square of body height $\left(\mathrm{m}^{2}\right)$. We also collected clinical information about disease duration from the diagnosis to enrolment (year), the interval between the initiation of the symptoms and the diagnosis (months), the length of morning stiffness (min), and coexisting diseases. The presence of bone erosions on the hands, feet, and other involved joints assessed by X-ray and extra-articular manifestations (involvement of skin, eye, respiratory, renal, nervous, and gastrointestinal systems) were also evaluated and recorded at the admission. Other hematological and cardiac complaints related to RA were also questioned.

The levels of erythrocyte sedimentation rate $(\mathrm{mm} / \mathrm{h})$, C-reactive protein (CRP, $\mathrm{mg} / \mathrm{l})$, immunoglobulin-M rheumatoid factor (RF), and anti-cyclic citrullinated peptide antibody (ACPA) at the initiation of the registry were measured. Depending on the laboratory upper limits of each institution, we grouped the patients as positive (higher than the upper level of laboratory range) and negative for RF and ACPA. 
We reviewed the erosive status on X-rays of the joints. We performed the baseline ultrasonographic examination of the DAS 28 joints as defined previously in a limited number of patients [8].

The details of current and previous medical treatment, including the name of the drugs, dosages, duration, and switching status, were obtained and recorded. Clinical information, including tender and swollen joint counts in 28 joints $[6,10]$, patient's and physician's global visual analogue scale (VAS), VAS scores of pain and fatigue, and the Health Assessment Questionnaire (HAQ) Disability Index [13, 14] were collected during the admission to the registry.

After enrollment, the patients were examined every 6 months for physical examination, compliance to the medical treatment, and monitoring the clinical response. The treating physicians were responsible for treatment decisions, including bDMARD or tsDMARD selection, co-medications, dosage adjustments, or switches based on the clinical assessments or patient's preferences [14].

\section{Clinical Assessment of Disease Activity}

We used the Boolean criteria for the disease activity and remission [14, 15]. Tender joint score $\leq 1$, swollen joint score $\leq 1$, and normal CRP $(\leq 1 \mathrm{mg} / \mathrm{dl})$ were regarded as remission.

Besides, Erythrocyte Sedimentation Ratebased Disease Activity Score in 28 joints (DAS28-ESR), C-reactive protein-based Disease Activity Score in 28 joints (DAS28-CRP), Clinical Disease Activity Index (CDAI) score, and the Simplified Disease Activity Index (SDAI, $0-86)$ were also used to grade the disease $[2,5,6,16]$.

The scores of DAS28-ESR and DAS-CRP $<2.6$ according to the EULAR criteria were regarded as remission $[3,17]$. Disease activity was defined as low $(\leq 3.2)$, moderate (between $<3.2$ and $\leq 5.1)$, or high $(>5.1)$ based on the scores DAS28-ESR and DAS-CRP [3].

The cut-off scores of CDAI were as follows: remission $(<2.8)$, low $(\geq 2.8$ and $\leq 10.0)$, moderate $(>10.0$ and $\leq 22.0)$, and high activities $(>22.0)$ [16].
Tender and swollen joint counts in 28 joints, patient's and physician's global VAS, and CRP level are calculated to find the numerical sum of the SDAI $[15,18]$. The SDAI was used to assess the outcome, and we used the cut-off values of $\geq 3.3$ after 6 months to define the situation of non-remission, including low $(\geq 3.3$ and $\leq 11.0)$, moderate $(>11.0$ and $\leq 26.0)$, and high activities ( $>26.0)$.

\section{Groups}

The patients were regarded as the initial group within the first 6 months of the biological/targeted synthetic treatment (Group I). The patients with the first bDMARD-tsDMARD who had been continuing the same treatment for at least 6 months were defined as the sustained treatment group (Group ST). In the presence of any switching history of bDMARD or tsDMARD to another therapy, these patients were grouped as the switch group (Group S). The patients in Group I did not complete the first sixth month of advanced treatment with either bDMARDs or tsDMARDs. Thus, these patients were excluded from the comparative analysis between the patients with sustained treatment (Group ST) and drug switching (Group S).

\section{Statistical Analysis}

The primary objective of this study was to determine the proportion of patients who maintained sustained biologic/targeted synthetic treatment without any drug switching and compare the demographic and clinical characteristics of RA patients in Groups $S$ and ST. The secondary objective was to describe the baseline parameters of all RA patients in Groups I, S, and ST. All categorical variables were summarized with frequencies and percentages. In contrast, the numerical variables were summarized with mean and standard deviations (SD) or medians and interquartile ranges (Q1, Q3) according to their distributions in addition to minimum and maximum values. The normality assumption was assessed with the Kolmogorov-Smirnov test in addition to visual inspection by Q-Q and PP plots. The 
Table 1 Demographic characteristics of the overall patients and the patients in the study groups

\begin{tabular}{|c|c|c|c|c|c|c|}
\hline & & $\begin{array}{l}\text { Overall } \\
(n=565)\end{array}$ & $\begin{array}{l}\text { Group I } \\
(n=104)\end{array}$ & $\begin{array}{l}\text { Group ST } \\
(n=267)\end{array}$ & $\begin{array}{l}\text { Group S } \\
(n=194)\end{array}$ & $p^{*}$ \\
\hline Age (years) ${ }^{a}$ & & $53.7 \pm 12.8$ & $53.1 \pm 12.8$ & $53.9 \pm 13.6$ & $53.7 \pm 11.7$ & 0.534 \\
\hline \multirow[t]{2}{*}{ Sex $*$} & Male & $111(19.6)$ & $25(24.0)$ & $62(23.2)$ & $24(12.4)$ & 0.003 \\
\hline & Female & $454(80.4)$ & $79(76.0)$ & $205(76.8)$ & $170(87.6)$ & \\
\hline BMI $\left(\mathrm{kg} / \mathrm{m}^{2}\right)^{\mathrm{a}}$ & & $28.1 \pm 5.3$ & $27.2 \pm 5.3$ & $28.0 \pm 5.2$ & $28.7 \pm 5.6$ & 0.317 \\
\hline \multirow[t]{4}{*}{ Smoking status ${ }^{\mathrm{b}}$} & Active & $78(13.8)$ & $26(25.0)$ & $32(12.0)$ & $19(9.8)$ & 0.483 \\
\hline & Ex-smoker & $75(13.3)$ & $14(13.5)$ & $35(13.1)$ & $26(13.4)$ & \\
\hline & Non-smoker & $407(72.0)$ & $63(60.6)$ & $198(74.2)$ & $146(75.3)$ & \\
\hline & Unknown & $5(0.9)$ & $1(1.0)$ & $2(0.7)$ & $3(1.5)$ & \\
\hline \multirow[t]{4}{*}{ Alcohol status ${ }^{\mathrm{b}}$} & Active & $15(2.7)$ & $6(4.8)$ & $7(2.6)$ & $3(1.5)$ & 0.750 \\
\hline & Ex-drinker & $5(0.9)$ & $2(1.6)$ & $3(1.1)$ & $1(0.5)$ & \\
\hline & Non-drinker & $53.3(94.5)$ & $113(89.7)$ & $251(94.0)$ & $187(96.4)$ & \\
\hline & Unknown & $11(2.0)$ & $5(4.0)$ & $6(2.2)$ & $3(1.5)$ & \\
\hline \multirow[t]{2}{*}{ Marital status ${ }^{\mathrm{b}}$} & Single & $82(14.5)$ & $24(23.1)$ & $35(13.1)$ & $23(11.9)$ & 0.689 \\
\hline & Married & $483(85.5)$ & $80(76.9)$ & $232(86.9)$ & $171(88.1)$ & \\
\hline $\begin{array}{c}\text { Number of } \\
\text { children }^{c}\end{array}$ & & $2(2,4)$ & $2(1,3)$ & $3(2,4)$ & $3(2,4)$ & 0.788 \\
\hline \multirow{3}{*}{$\begin{array}{l}\text { Educational } \\
\text { status }^{b}\end{array}$} & Primary/illiterate & $366(64.8)$ & $63(60.6)$ & $175(65.5)$ & $128(66.0)$ & 0.930 \\
\hline & Secondary/college & $162(28.7)$ & $8(7.7)$ & $16(6.0)$ & $13(6.7)$ & \\
\hline & $\begin{array}{l}\text { University or } \\
\text { higher }\end{array}$ & $37(6.5)$ & $33(31.7)$ & $76(28.5)$ & $53(27.3)$ & \\
\hline \multirow{8}{*}{$\begin{array}{l}\text { Geographical } \\
\text { regions }^{b}\end{array}$} & Black Sea & $59(10.4)$ & $6(5.8)$ & 37 (13.9) & $16(8.2)$ & 0.017 \\
\hline & Marmara & $100(17.7)$ & $12(11.5)$ & $45(16.9)$ & $43(22.2)$ & \\
\hline & Aegean & $75(13.3)$ & $18(17.3)$ & $39(14.6)$ & $18(9.3)$ & \\
\hline & Mediterranean & $73(12.9)$ & $45(43.3)$ & $21(7.9)$ & $7(3.6)$ & \\
\hline & Central Anatolia & $118(20.9)$ & $12(11.5)$ & $63(23.6)$ & $43(22.2)$ & \\
\hline & Eastern Anatolia & $92(16.3)$ & $11(10.6)$ & $36(13.5)$ & $45(23.2)$ & \\
\hline & $\begin{array}{c}\text { Southeastern } \\
\text { Anatolia }\end{array}$ & $46(8.1)$ & $0(0.0)$ & $25(9.4)$ & $21(10.8)$ & \\
\hline & Others & $2(0.4)$ & 0 & 1 & 1 & \\
\hline
\end{tabular}

${ }^{\mathrm{a}}:$ Mean \pm standard deviation, ${ }^{\mathrm{b}}: n(\%),{ }^{\mathrm{c}}:$ median (IQR1, IQR3). ${ }^{*}:$ between Group ST and Group $S$

$I$ Initial, $S T$ sustained treatment, $S$ switch, $B M I$ body mass index 
Table 2 Clinical characteristics of the overall patients and the patients in the study groups

\begin{tabular}{|c|c|c|c|c|c|c|}
\hline & & $\begin{array}{l}\text { Overall } \\
(n=565)\end{array}$ & $\begin{array}{l}\text { Group I } \\
(n=104)\end{array}$ & $\begin{array}{l}\text { Group ST } \\
(n=267)\end{array}$ & $\begin{array}{l}\text { Group } S \\
(n=194)\end{array}$ & $p^{*}$ \\
\hline Disease duration (years) ${ }^{b}$ & & $10(5,15)$ & $5(2,9)$ & $10(6,15.5)$ & $11(8,17)$ & 0.002 \\
\hline Delay for diagnosis (years) ${ }^{b}$ & & $1(0,2)$ & $0(0,1)$ & $1(0,2)$ & $1(0,3)$ & 0.717 \\
\hline \multirow[t]{9}{*}{ Coexisting disease $^{a}$} & & $274(48.5)$ & $47(45.2)$ & $135(50.6)$ & $92(47.4)$ & 0.506 \\
\hline & Hypertension & $165(30.1)$ & $29(28.7)$ & $85(31.8)$ & $51(27.0)$ & 0.176 \\
\hline & $\begin{array}{l}\text { Diabetes } \\
\text { mellitus }\end{array}$ & $85(15.8)$ & $16(16.3)$ & $42(15.7)$ & $27(14.4)$ & 0.511 \\
\hline & Renal diseases & $16(3.1)$ & $2(2.3)$ & $9(3.4)$ & $5(2.7)$ & 0.544 \\
\hline & Hyperlipidemia & $53(12.5)$ & $9(13.0)$ & $26(9.7)$ & $18(15.7)$ & 0.981 \\
\hline & COPD & $32(5.7)$ & $3(2.9)$ & $21(7.9)$ & $8(4.1)$ & 0.102 \\
\hline & $\begin{array}{l}\text { Cardiovascular } \\
\text { diseases }\end{array}$ & $45(8.9)$ & $8(8.5)$ & $26(9.7)$ & $11(6.2)$ & 0.086 \\
\hline & Cancer & $10(1.9)$ & $4(4.2)$ & $6(2.2)$ & $0(0.0)$ & 0.034 \\
\hline & $\begin{array}{l}\text { Thyroid } \\
\text { problems }\end{array}$ & $64(12.7)$ & $13(14.4)$ & $24(9.0)$ & $27(15.6)$ & 0.087 \\
\hline $\begin{array}{l}\text { Multiple comorbidities } \\
\qquad(\geq 2)^{\mathrm{a}}\end{array}$ & & $81(14.3)$ & $14(13.5)$ & $45(16.9)$ & $22(11.3)$ & 0.097 \\
\hline Erosive arthritis ${ }^{\mathrm{a}}$ & & $288(51.0)$ & $43(41.3)$ & $128(47.9)$ & $117(60.3)$ & 0.009 \\
\hline \multicolumn{7}{|l|}{$\begin{array}{l}\text { Extra-articular } \\
\text { manifestations }\end{array}$} \\
\hline \multirow{7}{*}{$\begin{array}{l}\text { Types of extra-articular } \\
\text { involvement }^{\text {a }}\end{array}$} & Respiratory & $59(10.4)$ & $9(8.7)$ & $29(10.9)$ & $21(10.8)$ & 0.990 \\
\hline & Skin & $25(4.4)$ & $2(1.9)$ & $11(4.1)$ & $12(6.2)$ & 0.315 \\
\hline & Ocular & $13(12.5)$ & $80(14.2)$ & $35(13.1)$ & $32(16.5)$ & 0.308 \\
\hline & Hematological & $132(23.4)$ & $35(33.7)$ & $42(15.7)$ & $55(28.4)$ & 0.001 \\
\hline & Neurological & $7(6.7)$ & $40(7.1)$ & $3(1.1)$ & $0(0.0)$ & 0.139 \\
\hline & Cardiac & $3(0.5)$ & $0(0.0)$ & $23(8.6)$ & $10(5.2)$ & 0.155 \\
\hline & Osteoporosis & $26(4.6)$ & $3(2.9)$ & $13(4.9)$ & $10(5.2)$ & 0.889 \\
\hline \multirow[t]{3}{*}{$\mathrm{RF}^{\mathrm{a}}$} & Negative & $86(19.6)$ & $16(20.0)$ & $45(21.4)$ & $25(16.8)$ & 0.273 \\
\hline & Positive & $353(80.4)$ & $64(80.0)$ & $165(78.6)$ & $124(83.2)$ & \\
\hline & Unknown & 126 & 24 & 57 & 45 & \\
\hline \multirow[t]{3}{*}{$\mathrm{ACPA}^{\mathrm{a}}$} & Negative & $113(26.3)$ & $18(22.0)$ & $52(25.1)$ & $43(30.5)$ & 0.269 \\
\hline & Positive & $317(73.7)$ & $64(78.0)$ & $155(74.9)$ & $98(69.5)$ & \\
\hline & Unknown & 135 & 24 & 60 & 53 & \\
\hline
\end{tabular}


Table 2 continued

\begin{tabular}{|c|c|c|c|c|c|c|}
\hline & & $\begin{array}{l}\text { Overall } \\
(n=565)\end{array}$ & $\begin{array}{l}\text { Group I } \\
(n=104)\end{array}$ & $\begin{array}{l}\text { Group ST } \\
(n=267)\end{array}$ & $\begin{array}{l}\text { Group } S \\
(n=194)\end{array}$ & $p^{*}$ \\
\hline \multirow[t]{2}{*}{ Morning stiffness $(\mathrm{min})^{\mathrm{b}}$} & & $10(0,30)$ & $10(0,30)$ & $10(0,20)$ & $10(0,30)$ & 0.223 \\
\hline & Missing & 69 & 10 & 32 & 27 & \\
\hline Tender joint score ${ }^{\mathrm{b}}$ & & $0(0,4)$ & $0(0,4)$ & $0(0,3)$ & $1(0,4)$ & 0.064 \\
\hline Swollen joint score ${ }^{\mathrm{b}}$ & & $0(0,0)$ & $0(0,0)$ & $0(0,0)$ & $0(0,0)$ & 0.040 \\
\hline
\end{tabular}

homogeneity of variance was assessed using Levene's test. The numerical variables were analyzed across two groups with Mann-Whitney $U$ test or $t$ test. The Chi-square test was used to compare categorical variables. A $p$ value smaller than 0.05 represented the statistical significance. The R version 4.0.4 (2021-02-15) was employed for all analyses.

\section{RESULTS}

The study included a total of 565 patients. There were 104, 267, and 194 patients in Groups I, ST, and S, respectively. Considering all patients, the mean age was $53.7 \pm 12.8$ years, and 454 females (80.4\%) and 111 males (19.6\%) were enrolled. The demographic features are given in Table 1 . The majority of the patients were non-smokers (72.0\%) and non-drinkers (94.5\%). The Central Anatolia and Marmara regions were the most frequent geographical areas where the patients lived (20.9 and $17.7 \%)$, followed by Eastern Anatolia (16.3\%).

The median disease duration was 10 years. Hypertension and diabetes mellitus were the most frequent comorbidities seen in 165 $(30.1 \%)$ and 85 patients (15.8\%). In 81 patients $(14.3 \%)$, there were multiple comorbidities $(\geq 2)$. The rates of positivity for RF and ACPA were 80.4 and $73.7 \%$. The clinical characteristics of all patients are given in Table 2 .

Based on the Boolean remission criteria, most of the patients $(99.1 \%)$ were in non-remission status. The median DAS28-ESR and DAS28-CRP scores were 3.1 and 3.0 in the overall group. According to DAS28-ESR and DAS28-CRP, 34.2 and $35.8 \%$ of the cases were in remission. However, 12.1 and $6.4 \%$ of the patients had reached CDAI and SDAI remission. The classification of the patients according to the disease activity scoring systems is given in Table 3.

The demographic and clinical characteristics of the patients in Group I are summarized in Tables 1, 2, and 3. The mean age was $53.1 \pm 12.8$ years. The median length of disease duration in Group I was 5 years. Most of the patients were positive for RF (80.0\%) and ACPA (78.0\%). The disease activity scores of the patients in Group I are given in Table 3. We calculated the median scores of the DAS28-ESR and DAS28-CRP as 3.1 and 3.2. According to the Boolean remission system, although most patients $(n=97)$ were in non-remission status, 33.0 and $32.7 \%$ were regarded as remission based on the DAS28-ESR and DAS28-CRP. Based on the CDAI, LDA was the most common group seen in 33 patients $(37.1 \%)$, but we detected moderate disease activity (MDA) as the most common disease activity (44\%) considering SDAI.

There were 267 patients in Group ST and 194 patients in Group S (Table 1). The groups were similar in age, BMI, cigarette and alcohol consumption, and educational and marital status (Table 1). In Group S, the rate of female patients was significantly higher than the Group ST ( 87.6 vs. $76.8 \%, p=0.003$ ). There were significant differences in the distribution of the 
Table 3 Evaluation of disease activity scoring in the overall patients and the patients in the study groups

\begin{tabular}{|c|c|c|c|c|c|c|}
\hline & & $\begin{array}{l}\text { Overall } \\
(n=565)\end{array}$ & $\begin{array}{l}\text { Group I } \\
(n=104)\end{array}$ & $\begin{array}{l}\text { Group ST } \\
(n=267)\end{array}$ & $\begin{array}{l}\text { Group S } \\
(n=194)\end{array}$ & $p^{*}$ \\
\hline \multirow{2}{*}{$\begin{array}{l}\text { Boolean non- } \\
\text { remission }^{\mathrm{a}}\end{array}$} & & $525(99.1)$ & $97(100.0)$ & $249(97.3)$ & $179(99.4)$ & 0.325 \\
\hline & Missing & 35 & 7 & 14 & 14 & \\
\hline DAS28-ESR ${ }^{b}$ & & $3.1(2.3,4.2)$ & $3.1(2.4,4.3)$ & $3.1(2.3,4.0)$ & $3.3(2.3,4.4)$ & 0.147 \\
\hline \multirow{5}{*}{$\begin{array}{l}\text { DAS28-ESR } \\
\text { groups }^{a}\end{array}$} & Remission $(<2.6)$ & $178(34.2)$ & $31(33.0)$ & $91(36.8)$ & $56(31.1)$ & 0.476 \\
\hline & $\mathrm{LDA}(\geq 2.6, \leq 3.2)$ & $101(19.4)$ & $24(25.5)$ & $44(17.8)$ & $33(18.3)$ & \\
\hline & $\operatorname{MDA}(>3.2, \leq 5.1)$ & $166(31.9)$ & $21(22.3)$ & $83(33.6)$ & $62(34.4)$ & \\
\hline & $\mathrm{HDA}(>5.1)$ & $76(14.6)$ & $18(19.1)$ & $29(11.7)$ & $29(16.1)$ & \\
\hline & Missing & 44 & 10 & 20 & 14 & \\
\hline DAS28-CRP ${ }^{b}$ & & $3.0(2.4,4.0)$ & $3.2(2.5,4.4)$ & $2.9(2.4,3.7)$ & $3.2(2.3,4.2)$ & 0.025 \\
\hline \multirow{5}{*}{$\begin{array}{l}\text { DAS28-CRP } \\
\text { groups }^{a}\end{array}$} & Remission $(<2.6)$ & $197(35.8)$ & $33(32.7)$ & $98(37.4)$ & $66(35.1)$ & 0.018 \\
\hline & $\mathrm{LDA}(\geq 2.6, \leq 3.2)$ & $114(20.7)$ & $20(19.8)$ & $66(25.2)$ & $28(14.9)$ & \\
\hline & $\operatorname{MDA}(>3.2, \leq 5.1)$ & $191(34.7)$ & $31(30.7)$ & $83(31.7)$ & $77(41.0)$ & \\
\hline & $\operatorname{HDA}(>5.1)$ & $49(8.9)$ & $17(16.8)$ & $15(5.7)$ & $17(9.0)$ & \\
\hline & Missing & 14 & 3 & 5 & 6 & \\
\hline $\mathrm{CDAI}^{\mathrm{b}}$ & & $10(6,17)$ & $11(6,19)$ & $10(5,15)$ & $12(6,18)$ & 0.010 \\
\hline \multirow[t]{5}{*}{ CDAI groups ${ }^{\mathrm{a}}$} & Remission $(<2.8)$ & $61(12.1)$ & $11(12.4)$ & $28(11.8)$ & $22(12.3)$ & $<0.001$ \\
\hline & $\begin{array}{l}\mathrm{LDA} \\
\qquad(\geq 2.8, \leq 10.0)\end{array}$ & $201(39.6)$ & $33(37.1)$ & $114(48.1)$ & $53(29.6)$ & \\
\hline & $\begin{array}{l}\text { MDA } \\
\qquad(>10.0, \leq 22.0)\end{array}$ & $178(34.5)$ & $27(30.3)$ & $73(30.8)$ & $74(41.3)$ & \\
\hline & $\mathrm{HDA}(>22.0)$ & $70(13.9)$ & $18(20.2)$ & $22(9.3)$ & $30(16.8)$ & \\
\hline & Missing & 60 & 15 & 30 & 15 & \\
\hline $\mathrm{SDAI}^{\mathrm{b}}$ & & $12.4(7.3,19)$ & $\begin{array}{c}14.2(8.7 \\
21.9)\end{array}$ & $11(7,17.6)$ & $13.7(8.3,20.0)$ & 0.003 \\
\hline \multirow[t]{5}{*}{ SDAI groups ${ }^{a}$} & Remission $(<3.3)$ & $35(6.4)$ & $7(7.0)$ & $18(7.0)$ & $10(5.4)$ & 0.005 \\
\hline & $\begin{array}{l}\text { LDA } \\
\qquad(\geq 3.3, \leq 11.0)\end{array}$ & $195(35.8)$ & $31(31.0)$ & $111(43.0)$ & $53(28.5)$ & \\
\hline & $\begin{array}{l}\text { MDA } \\
\qquad(>11.0, \leq 26.0)\end{array}$ & $250(46.0)$ & $44(44.0)$ & $109(42.2)$ & $97(52.2)$ & \\
\hline & $\mathrm{HDA}(>26.0)$ & $64(11.8)$ & $18(18.0)$ & $20(7.8)$ & $26(14.0)$ & \\
\hline & Missing & 21 & 4 & 9 & 8 & \\
\hline
\end{tabular}


Table 3 continued

\begin{tabular}{llllllr}
\hline & $\begin{array}{l}\text { Overall } \\
(\boldsymbol{n}=\mathbf{5 6 5})\end{array}$ & $\begin{array}{l}\text { Group I } \\
(\boldsymbol{n}=\mathbf{1 0 4})\end{array}$ & $\begin{array}{l}\text { Group ST } \\
(\boldsymbol{n}=\mathbf{2 6 7})\end{array}$ & $\begin{array}{l}\text { Group S } \\
(\boldsymbol{n}=\mathbf{1 9 4})\end{array}$ & $\boldsymbol{p}^{*}$ \\
\hline VAS $^{\mathrm{b}}$ & $\begin{array}{c}\text { Patient's global } \\
\text { assessment }\end{array}$ & $4(2,5)$ & $5(2,6)$ & $4(2,5)$ & $5(3,6.3)$ & $<0.001$ \\
& $\begin{array}{l}\text { Physician's global } \\
\text { assessment }\end{array}$ & $4(2,5)$ & $5(2,6)$ & $3(2,5)$ & $5(3,6)$ & $<0.001$ \\
& $\begin{array}{l}\text { Pain } \\
\text { Fatigue }\end{array}$ & $4(2,6)$ & $5(2,6.3)$ & $3(2,5)$ & $5(3,6.6)$ & $<0.001$ \\
& $5(3,6)$ & $5(2,6)$ & $5(3,6)$ & $5(3,7)$ & 0.008 \\
$\mathrm{HAQ}^{\mathrm{b}}$ & & $0.55(0.1,1.1)$ & $0.4(0.1,0.9)$ & $0.5(0.05,1)$ & $0.7(0.2,1.2)$ & 0.015 \\
& 14 & 3 & 5 & 6 & \\
\hline
\end{tabular}

${ }^{\mathrm{a}}: n(\%),{ }^{\mathrm{b}}:$ median (IQR1, IQR3). ${ }^{*}:$ between Group ST and Group $\mathrm{S}$

$I$ initial, $S T$ sustained treatment, $S$ switch, DAS28-ESR The Disease Activity Score-28 for Rheumatoid Arthritis with ESR, $L D A$ low disease activity, $M D A$ moderate disease activity, $H D A$ high disease activity, $D A S 28$-CRP The Disease Activity Score-28 for Rheumatoid Arthritis with C-reactive protein, $C D A I$ clinical disease activity, $S D A I$ simple disease activity index, VAS Visual Analog Scale, HAQ health assessment questionnaire

Table 4 Treatment details

\begin{tabular}{|c|c|c|c|c|c|c|}
\hline Medication/group & & $\begin{array}{l}\text { Overall } \\
(n=565)\end{array}$ & $\begin{array}{l}\text { Group I } \\
(n=104)\end{array}$ & $\begin{array}{l}\text { Group ST } \\
(n=267)\end{array}$ & $\begin{array}{l}\text { Group } S \\
(n=194)\end{array}$ & $p^{*}$ \\
\hline $\begin{array}{l}\text { Concurrent NSAID }{ }^{\mathrm{a}} \\
n(\%)\end{array}$ & & $70(12.5)$ & $18(17.5)$ & $27(10.1)$ & $25(13.2)$ & 0.312 \\
\hline $\begin{array}{l}\text { Concurrent } \\
\text { glucocorticoid }^{\mathrm{a}} n(\%)\end{array}$ & & $184(32.9)$ & $34(32.7)$ & $82(31.8)$ & $68(35.6)$ & 0.296 \\
\hline Monotherapy $^{\mathrm{a}} n(\%)$ & & $38(6.7)$ & $18(17.3)$ & $20(7.8)$ & $0(0.0)$ & $<0.001$ \\
\hline \multirow{4}{*}{$\begin{array}{l}\text { Combined therapy } \\
n(\%)\end{array}$} & & $527(93.3)$ & $86(82.7)$ & 247 (95.7) & $194(100.0)$ & $<0.001$ \\
\hline & csDMARDs & $20(6.7)$ & $15(34.1)$ & $2(1.3)$ & $3(2.8)$ & $<0.001$ \\
\hline & $\begin{array}{l}\text { csDMARD- } \\
\text { bDMARD }\end{array}$ & $247(82.6$ & $25(56.8)$ & $129(86.6)$ & $93(87.7)$ & $<0.001$ \\
\hline & $\begin{array}{l}\text { csDMARD- } \\
\text { tsDMARD }\end{array}$ & $32(10.7)$ & $4(9.1)$ & $18(12.1)$ & $10(9.4)$ & 0.598 \\
\hline
\end{tabular}

$\stackrel{a}{a}: n(\%),{ }^{*}:$ between Group ST and Group $S$

$I$ initial, $S T$ sustained treatment, $S$ switch, NSAID non-steroid anti-inflammatory drugs, cs conventional synthetic, DMARD disease-modifying antirheumatic drug, $b$ biological, $t$ s targeted synthetic 
Table 5 Distribution of the medications

\begin{tabular}{|c|c|c|c|c|c|c|c|}
\hline Drug group & & Medication & Status & $\begin{array}{l}\text { Overall } \\
(n=565)\end{array}$ & $\begin{array}{l}\text { Group I } \\
(n=104)\end{array}$ & $\begin{array}{l}\text { Group ST } \\
(n=267)\end{array}$ & $\begin{array}{l}\text { Group S } \\
(n=194)\end{array}$ \\
\hline \multirow[t]{8}{*}{ csDMARD } & & Methotrexate & $\begin{array}{r}\text { Active } \\
\text { user }\end{array}$ & $182(32.2)$ & 34 & 88 & 60 \\
\hline & & & Ex-user & $297(52.6)$ & 49 & 135 & 118 \\
\hline & & Hydroxychloroquine & $\begin{array}{r}\text { Active } \\
\text { user }\end{array}$ & $79(14.0)$ & 24 & 32 & 23 \\
\hline & & & Ex-user & $201(35.6)$ & 27 & 83 & 91 \\
\hline & & Leflunomide & $\begin{array}{r}\text { Active } \\
\text { user }\end{array}$ & $139(24.6)$ & 38 & 55 & 46 \\
\hline & & & Ex-user & $152(26.9)$ & 15 & 68 & 69 \\
\hline & & Sulfasalazine & $\begin{array}{r}\text { Active } \\
\text { user }\end{array}$ & $17(3.0)$ & 7 & 5 & 5 \\
\hline & & & Ex-user & $195(34.5)$ & 23 & 90 & 82 \\
\hline \multicolumn{8}{|l|}{$\mathrm{b} / \mathrm{ts}$} \\
\hline \multicolumn{8}{|l|}{ DMARDS } \\
\hline & \multirow[t]{10}{*}{$\begin{array}{l}\text { TNF } \\
\text { inhibitors }\end{array}$} & Adalimumab & $\begin{array}{r}\text { Active } \\
\text { user }\end{array}$ & $62(11.0)$ & 8 & 38 & 16 \\
\hline & & & Ex-user & $74(13.1)$ & 2 & 3 & 69 \\
\hline & & Etanercept & $\begin{array}{r}\text { Active } \\
\text { user }\end{array}$ & $73(12.9)$ & 4 & 57 & 12 \\
\hline & & & Ex-user & $81(14.3)$ & 2 & 1 & 78 \\
\hline & & Certolizumab pegol & $\begin{array}{r}\text { Active } \\
\text { user }\end{array}$ & $17(3.0)$ & 0 & 6 & 11 \\
\hline & & & Ex-user & $8(1.4)$ & 1 & 1 & 6 \\
\hline & & Infliximab & $\begin{array}{r}\text { Active } \\
\text { user }\end{array}$ & $22(3.9)$ & 2 & 14 & 6 \\
\hline & & & Ex-user & $29(5.1)$ & 0 & 0 & 29 \\
\hline & & Golimumab & $\begin{array}{r}\text { Active } \\
\text { user }\end{array}$ & $34(6.0)$ & 7 & 20 & 7 \\
\hline & & & Ex-user & $28(5.0)$ & 0 & 1 & 27 \\
\hline & \multirow[t]{4}{*}{$\begin{array}{l}\text { Non-TNF } \\
\text { inhibitors }\end{array}$} & Tocilizumab & $\begin{array}{r}\text { Active } \\
\text { user }\end{array}$ & $85(15.0)$ & 9 & 26 & 50 \\
\hline & & & Ex-user & $20(3.5)$ & 0 & 1 & 19 \\
\hline & & Abatacept & $\begin{array}{r}\text { Active } \\
\text { user }\end{array}$ & $22(3.9)$ & 0 & 13 & 9 \\
\hline & & & Ex-user & $36(6.4)$ & 0 & 2 & 34 \\
\hline
\end{tabular}


Table 5 continued

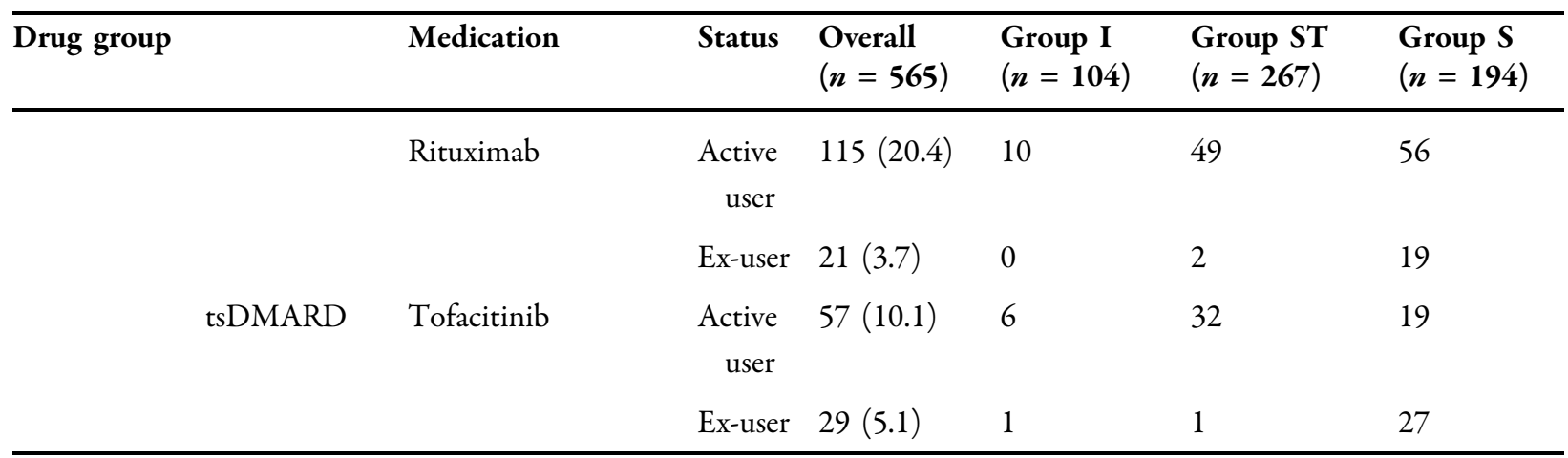

cs conventional synthetic, DMARD disease-modifying antirheumatic drugs, $b$ biological, $t s$ targeted synthetic, TNF tumor necrosis factor

geographical regions between the groups $(p=0.017)$.

The median disease duration was 10 years in Group ST and 11 years in Group S. The difference in the median disease duration between the groups (ST and S) was significant $(p=0.002)$. Erosive arthritis was more frequently detected in patients with Group $S$ than the Group ST $(p=0.009)$. There were significantly more patients with hematological extraarticular involvement in Group $S$ than the Group ST $(p=0.001)$. The clinical characteristics of the study groups are summarized in Table 2.

Table 3 presents the results of the disease activity scoring systems. The median scores of the DAS28-CRP, CDAI, and SDAI were significantly higher in Group S $(p=0.025, p=0.010$, and $p=0.003)$. According to the different disease activity scoring systems, rates of the patients with LDA and MDA were significantly higher in Group ST and Group S (Table 3). We also detected significant differences in the disease activity groups based on these scoring systems (Table 3). The median VAS scores of the patient's global, physician's global, pain, and fatigue were significantly higher in Group $S$ $(p<0.05)$. The HAQ score was significantly higher in Group S than the Group ST (0.7 vs. $0.5, p=0.015)$.

The treatment details of the medications and the different treatment modalities as monotherapy and combined therapy are detailed in Table 4 . The use of monotherapy was significantly higher in Group I $(p<0.001)$. Although $7.8 \%$ of the patients in the Group ST used monotherapy, there were no patients on monotherapy in Group $S(p<0.001)$. There were significant differences in the different drug combinations. The csDMARD medications were more frequently prescribed for the patients in Group I $(p<0.001)$. However, the patients in Groups ST and S used the csDMARD-bDMARD medications at a significantly higher rate than Group I $(p<0.001)$. The details of the medications considering the overall patients $(n=565)$ are summarized in Table 5.

\section{DISCUSSION}

This study was a multicenter, observational cross-sectional study using data from the BioStaR-RA registry in Turkey. It is one of the most comprehensive databases for RA in the country. In this study, we have found that despite using highly effective drugs, there was an unacceptable portion of the patients in the moderate disease activity group $(42.2 \%$ in sustained therapy and $52.2 \%$ in switch groups). Furthermore, we have detected that the patients with sustained therapies were more frequently male; they had lower composite disease activity scores, lower hematologic manifestations, lower 
patient's and physicians' global assessment scores, better functional status, and lesser radiographic damage. Overall, rituximab was the most commonly used drug, followed by tocilizumab and anti-TNF agents. This may be different from the actual distribution of other European countries. However, the centers involved in the BioSTAR-RA registry are tertiary care hospitals that admit referrals of patients with inadequate response to several agents. Therefore, the predominancy of pharmacological agents such as rituximab and tocilizumab, which were paid by the health insurance system in case of anti-TNF failure in context of a more resistive disease, may be partially explained by the fact that study hospitals are tertiary centers dealing with patients referred from other centers. A retrospective analysis of data collected from the 12th Adelphi Real World Disease Specific Programme for RA has been presented in a recent study. The rate to use tocilizumab and rituximab was lower among individuals with first-line advanced therapy, reaching 20\% and $9 \%$ after two switching cycles. However, the rates were about $5 \%$ for each agent in the overall analyses [12].

Biologic registries are essential sources for real-life data and long-term safety results. There are several biologic registries publishing efficacy and safety data periodically. Some of them are the Swedish Rheumatology Registers, including the Biologics Register (ARTIS), British Society for Rheumatology Biologics Register (BSRBR), the Spanish Rheumatology Registers including the Biologics register (BIOBADASER), Consortium of Rheumatology Researchers of North America (CORRONA), and German RABBIT registry [19-24]. The real-life data from various registers with distinct patterns of comorbidity rates and different sociodemographic, genetic, and geographic factors provide significant insights and contributions to data from randomized controlled trials. The sociodemographic features of our cohort, including sex, disease duration, seropositivity rate, comorbidities, and smoking status, are similar to other registries. A wellknown fact is that middle to older-aged individuals with female predominance are the most frequent patient group affected by RA. Our findings were comparable to these well- established features of RA $[3,5,11,17,25,26]$. Ageing is essential for developing higher risks and hospitalizations associated with comorbidities $[25,27]$. The mean age of the patients and the distribution of the comorbid diseases in each group were similar in the current study as Bawazir's study [17]. Murray et al. [3] reported better outcomes and responses in male patients with RA and psoriatic arthritis. Similarly, we also detected a higher proportion of male patients in the Group ST. However, large-scale studies are required to clarify any possible underlying mechanisms between sex hormones and pronounced inflammatory arthritis.

In a recent retrospective cohort study that analyzed treatment patterns in RA patients who were newly initiated on biologic or nonbiologic therapies within the US Corrona RA registry, switching was defined as the initiation of either a biologic or csDMARD therapy other than the discontinued agent. In this study, 70\% of the patients who switched their index therapy were more likely to have a BMI of $\geq 25$. The most common reason for the switch was reported as lack of efficacy. We have not observed a difference in BMI between sustained and switch therapy groups. The authors have concluded that switching from one therapy to another was common in the Corrona RA registry [28]. The switching rate in our study was $34.4 \%$. They reported that an unneglectable portion of patients in that registry was commenced with an anti-TNF monotherapy. Contrariwise, most of the combination therapies in the present cohort were based on cs DMARD and biologic/targeted synthetic drugs both in sustained or switch therapy groups.

Seropositivity and the presence of extra-articular involvements are regarded as poor prognostic markers for RA. Previous studies reported different RF and ACPA positivity rates reaching $90 \%[7,11,27]$. In the current study, we have determined 80.4 and $73.7 \%$ of our cohort were positive for RF and ACPA, respectively. Since the groups in this study were defined based on the pattern and adherence to treatment modalities instead of disease severity, we could not detect significant differences in seropositivity rates and overall extra-articular manifestations. 
It has been accepted that almost $40 \%$ of the patients show extra-articular manifestations of the disease $[27,29,30]$. In this study, we did not calculate an overall rate for extra-articular manifestations. However, we found that hematological extra-articular manifestations were more common in Group S than the others, which may indirectly imply its association with higher and hardly controllable disease activity. The absence of follow-up examinations in this study may be a limiting factor in evaluating the reciprocal associations between several parameters. The clinical and prognostic importance of hematological extra-articular manifestations is another issue that requires attention.

Erosive arthritis leading to some joint deformity is another disease feature directly associated with the initiation of treatment. The erosive disease seen in younger patients is also a significant risk factor for refractoriness to the biological agents [31]. Previous studies reported the incidence of erosive arthritis in RA populations was ranged up to $86 \%$ $[3,5,7,8,27,29,31]$. In our study, the incidence was $51.0 \%$. Khaliq et al. (29) speculated that such deformities are rarely seen in Western countries secondary to the prompt diagnosis and treatment of the disease, contrary to the previously published studies $[3,5,8,31]$. We have difficulty in explaining the relatively lower incidences of erosive disease in Western countries. Such controversial findings may partially be elucidated by distinct genetic backgrounds of these countries or missing radiographic data of the studies. We detected significant differences in the length of disease duration, the incidences of erosive arthritis, and hematological manifestations between Group ST and Group S. Longer disease duration or higher incidence of erosive arthritis may be regarded as the reflections of higher disease activity that necessitates drug switching. It should be kept in mind that we grouped the patients based on the real-world data of the drug regimens. This issue may be considered while analyzing the causative relationships.

The remission rates based on the DAS28-CRP in the large-scale studies of RA are approximately $40 \%$, contrary to higher rates reported by others $[11,17,25,32]$. We found that $35.5 \%$ of all patients had disease remission. The rates were in a range from 32.7 to $37.4 \%$, depending on the groups. Early diagnosis and prompt management of RA have been reported as the modulating factors on the erosive and prevalent nature of the disease [17]. Almoallim et al. [11] proposed several explanations for their relatively higher rates of remission. They thought that higher rates of low disease activity upon entry, earlier detection strategies, sound referral systems, and easier access to biologics are potential causes. We think that the implementation of treat-to-target approaches is critical for reaching such improvements. However, according to our government's medical reimbursement system, biologic or synthetic DMARDs are permitted after the failure of at least three synthetic, conventional DMARDs in case of persistent high disease activity. We may speculate that these limitations in the reimbursement system cause these patients to lose their chance of optimal treatment, eliminating the access of patients with moderate disease activity to these drugs and thus their chance of remission.

Drug switching cannot be regarded as a direct clinical indicator of a more severe disease considering the study's design. However, significantly more patients were with MDA and high disease activity based on DAS28-CRP, CDAI, and SDAI scoring systems in Group S than Group ST. The association of these disease activity scoring systems with the remission status of RA has been shown previously [6]. Besides, the median HAQ score was 0.5 in the Group ST. It was significantly lower than Group $S$, indicating a possibly higher incidence of functional remission in Group ST [6]. These findings may be related to the inadequacy of the medical treatment or the presence of intractable patients in Group S. So, analysis of the registry outcomes with longer follow-up time is required to explain the possible associations between these parameters.

Several studies focused on maintaining sustained low disease activity or remission about different drug regimens $[16,33]$. Others presented their outcomes of RA patients on biologics using the follow-up data [10]. We did not evaluate the impact of the treatment on the 
remission rates and the switching on the outcome of the disease, which is already well known [34]. Instead, we have presented the data of patients on sustained versus switched therapies in a cross-sectional design. So, our results should be interpreted with the appreciation of study design. Several clinical factors such as female sex, older age, prolonged disease duration, higher baseline disease activity, tender joint counts, and more significant functional impairment have been negatively associated with sustained remission [34]. Similarly, patients with sustained therapies had a higher portion of male patients. In the current study, they had lower composite disease activity scores, lower hematologic manifestations, lower patient's and physicians' global assessment, better functional status, and less radiographic damage.

Although almost one-third of the patients were in remission according to the DAS 28-CRP, the remission rates may alter according to the disease activity scoring systems employed. Greenmyer et al. [35] found significant differences between the cut-off values of the DAS28ESR and DAS28-CRP for high disease activity and remission of RA. A novel study indicated that patients are inconsistently categorized into disease activity states using any two disease activity indices among DAS28-ESR, DAS28-CRP, SDAI, and CDAI with a disparity rate of 9.7-40.6\% [36]. They concluded that SDAI and CDAI agreed well while CDAI and SDAI did not establish a linear relationship with DAS28-CRP and DAS28-ESR and proposed a formula to convert values from one index to another. In this study, we have used several scoring systems and shown significant differences in the scores of DAS-28 CRP, CDAI, and SDAI between the groups. The correlation of these scoring systems with the severity of the disease activity was regarded as out of focus of the study.

In the current study, we have presented reallife data about RA based on the different therapeutic regimens, which is the major strength of this study. However, the study has some drawbacks. First of all, some radiographic data are missing, making the generalizability of the radiographic damage data harder. Also, it presents only descriptive and retrospective data and does not include drug compliance data of the individuals. Therefore, future studies on the cohort will provide more robust data after collecting higher prospective observations. The significant differences in the distribution of the groups between the different geographic regions of Turkey may affect the study's outcomes. However, there is uniform access for different treatment modalities of RA in all centers involved. Considering the cumulation of a vast majority of patients in the moderate disease activity group, it might have been more beneficial to sub-categorize patients with moderate disease activity further into low or high activity status as in the study by Genitsaridi et al. [10] due to the proposed heterogeneity within this group. In a recent study analyzing data from the Rheumatology Informatics System for Effectiveness, it was reported that oneto two-thirds of patients had failed to alter their treatments even if they had moderate or high disease activity [37]. Similar to their findings, in our cohort, the patients with moderate disease activity either in the switch or sustained groups, unfortunately, do not have the chance to change their treatments and reach low disease activity since the reimbursement system does not allow any drug switches to a similar or different mechanism of action unless high disease activity is available. Different applications of medical reimbursement and the requirements for drug switching between the Western countries and Turkey should be considered while evaluating our results. Another limitation may be that previous drug history depends on retrospective data either from patients' claims or each institution's medical records, which creates a bias resulting from recalling problems possible. Also, the centers involved in the registry are tertiary care centers, which impedes the generalizability of the results.

\section{CONCLUSIONS}

There is a conglomeration of low and moderate disease activity in both sustained and switch therapy groups, milder in the former. Lower disease activity scores, lower hematologic manifestations, better functional status, and 
less radiographic damage are associated with sustained treatment. Even though observational studies may not directly influence management strategies, we consider that our results are pivotal in presenting real-life data from an extensive registry. Follow-up studies of the BioSTaR registry will come up soon.

\section{ACKNOWLEDGEMENTS}

Funding. No funding or sponsorship was received for this study or publication of this article.

Authorship. All named authors meet the International Committee of Medical Journal Editors (ICMJE) criteria for authorship for this article, take responsibility for the integrity of the work as a whole, and have given their approval for this version to be published.

Author Contributions. Sebnem Ataman: Data acquisition, concept and design, drafting, statistical analysis, final approval of the version to be published. Ismihan Sunar: Data acquisition, drafting, final approval of the version to be published. Hatice Bodur: Data acquisition, concept and design, revising the content, final approval of the version to be published. Meltem Alkan Melikoglu: Data acquisition, critical revision, final approval of the version to be published. Hasan Fatih Cay: Data acquisition, critical revision, final approval of the version to be published. Erhan Capkin: Data acquisition, critical revision, final approval of the version to be published. Ozgur Akgul: Data acquisition, critical revision, final approval of the version to be published. Remzi Cevik: Data acquisition, critical revision, final approval of the version to be published. Feride Gogus: Data acquisition, critical revision, final approval of the version to be published. Ayhan Kamanli: Data acquisition, critical revision, final approval of the version to be published. Fatma Gul Yurdakul: Data acquisition, critical revision, final approval of the version to be published. Gulcan Gurer: Data acquisition, critical revision, final approval of the version to be published. Ilker Yagci: Data acquisition, critical revision, final approval of the version to be published. Aylin Rezvani: Data acquisition, critical revision, final approval of the version to be published. Mehmet Tuncay Duruoz: Data acquisition, critical revision, final approval of the version to be published. All authors agree to be accountable for all aspects of the work in ensuring that questions related to the accuracy or integrity of any part of the work.

Disclosures. Sebnem Ataman, Ismihan Sunar, Hatice Bodur, Meltem Alkan Melikoglu, Hasan Fatih Cay, Erhan Capkin, Ozgur Akgul, Remzi Cevik, Feride Gogus, Ayhan Kamanli, Fatma Gul Yurdakul, Gulcan Gurer, Ilker Yagci, Aylin Rezvani and Mehmet Tuncay Duruoz.

Compliance with Ethics Guidelines. The local ethical committee approved the study (Ankara Numune Training and Research Hospital, Number: E-182413; Turkish Medicine and Medical Devices Agency, 66175679-514.99E182413). This study was carried out in compliance with the Declaration of Helsinki. Written informed consent was taken from the participants.

Data Availability. The database underpinning our research is available in an electronic data repository. The datasets generated during and/or analyzed during the current study are available from the corresponding author on reasonable request.

\section{Declarations}

Conflict of interest The authors declare that they have no conflict of interest.

Open Access. This article is licensed under a Creative Commons Attribution-NonCommercial 4.0 International License, which permits any non-commercial use, sharing, adaptation, distribution and reproduction in any medium or format, as long as you give appropriate credit to the original author(s) and the source, provide a link to the Creative Commons licence, and indicate if changes were made. The images or other third party material in this article are included in the article's Creative Commons 
licence, unless indicated otherwise in a credit line to the material. If material is not included in the article's Creative Commons licence and your intended use is not permitted by statutory regulation or exceeds the permitted use, you will need to obtain permission directly from the copyright holder. To view a copy of this licence, visit http://creativecommons.org/licenses/by$\mathrm{nc} / 4.0 /$.

\section{REFERENCES}

1. Zhao J, Zhou W, Wu Y, Ji P, Yan X, Yang L, et al. FRI0143 The efficacy and cost-effectiveness of hydroxychloroquine, sulfasalazine, methotrexate triple therapy in preventing relapse of rheumatoid arthritis. Ann Rheum Dis. 2020;79(Suppl 1):655.

2. Vodencarevic A, Tascilar K, Hartmann F, Reiser M, Hueber AJ, Haschka J, et al. Advanced machine learning for predicting individual risk of flares in rheumatoid arthritis patients tapering biologic drugs. Arthritis Res Ther. 2021;23(1):67.

3. Murray K, Turk M, Alammari Y, Young F, Gallagher $\mathrm{P}$, Saber $\mathrm{T}$, et al. Long-term remission and biologic persistence rates: 12-year real-world data. Arthritis Res Ther. 2021;23(1):25.

4. Kameda H, Hirata A, Katagiri T, Takakura Y, Inoue $\mathrm{Y}$, Takenaka S, et al. Prediction of disease flare by biomarkers after discontinuing biologics in patients with rheumatoid arthritis achieving stringent remission. Sci Rep. 2021;11(1):6865.

5. Nekvindová L, Vencovský J, Pavelka K, Horák P, Křístková Z, Závada J. Switching first-line targeted therapy after not reaching low disease activity within 6 months is superior to conservative approach: a propensity score-matched analysis from the ATTRA registry. Arthritis Res Therapy [Internet]. 2021; 23(1):[11]. Available from: http:// europepmc.org/abstract/MED/33407803. Doi: https://doi.org/10.1186/s13075-020-02393-8. https://europepmc.org/articles/PMC7789592. https://europepmc.org/articles/PMC7789592?pdf= render

6. Koizumi R, Koyama K, Wako M, Ohba T, Takayama $\mathrm{Y}$, Haro H. Clinical conditions needed to acquire sustained functional remission in rheumatoid arthritis patients. Clin Rheumatol. 2021;40(5): 1751-7.

7. de Lucena Valim JM, Gonçalves Chaer FG, Guimarães da Silveira FD, da Silva ELVP, Batista de Souza BD. Switching of biological therapies in
Brazilian patients with rheumatoid arthritis. Future Sci OA. 2019;5(1):Fso355.

8. Vittecoq O, Desouches S, Kozyreff M, Nicolau J, Pouplin S, Rottenberg P, et al. Relapse in rheumatoid arthritis patients undergoing dose reduction and withdrawal of biologics: are predictable factors more relevant than predictive parameters? An observational prospective real-life study. BMJ Open. 2019;9(12):e031467.

9. Takanashi S, Kaneko Y, Takeuchi T. Characteristics of patients with difficult-to-treat rheumatoid arthritis in real-world. Rheumatology (Oxford). 2021. https://doi.org/10.1093/rheumatology/ keab209.

10. Genitsaridi I, Flouri I, Plexousakis D, Marias K, Boki $\mathrm{K}$, Skopouli F, et al. Rheumatoid arthritis patients on persistent moderate disease activity on biologics have adverse 5-year outcome compared to persistent low-remission status and represent a heterogeneous group. Arthritis Res Ther. 2020;22(1):226.

11. Almoallim $H$, Hassan $R$, Cheikh M, Faruqui $H$, Alquraa R, Eissa A, et al. Rheumatoid Arthritis Saudi Database (RASD): disease characteristics and remission rates in a tertiary care center. Open Access Rheumatol. 2020;12:139-45.

12 Holdsworth EA, Donaghy B, Fox KM, Desai P, Collier DH, Furst DE. Biologic and targeted synthetic DMARD utilization in the United States: Adelphi Real-World Disease-Specific Programme for Rheumatoid Arthritis. Rheumatol Therapy. 2021. https://doi.org/10.1007/s40744-021-00357-1.

13. Aletaha D, Neogi T, Silman AJ, Funovits J, Felson DT, Bingham CO 3rd, et al. Rheumatoid arthritis classification criteria: an American College of Rheumatology/European League Against Rheumatism collaborative initiative. Ann Rheum Dis. 2010;69(9):1580-8.

14. Abuhelwa AY, Hopkins AM, Sorich MJ, Proudman S, Foster DJR, Wiese MD. Association between obesity and remission in rheumatoid arthritis patients treated with disease-modifying anti-rheumatic drugs. Sci Rep [Internet]. 2020; 10(1):18634. Available from: http://europepmc.org/abstract/MED/ 33122725. Doi: https://doi.org/10.1038/s41598020-75673-7. https://europepmc.org/articles/ PMC7596471 https://europepmc.org/articles/ PMC7596471?pdf=render.

15. Felson DT, Smolen JS, Wells G, Zhang B, van Tuyl LH, Funovits J, et al. American College of Rheumatology/European League against Rheumatism provisional definition of remission in rheumatoid arthritis for clinical trials. Ann Rheum Dis. 2011;70(3):404-13. 
16. Combe B, Allanore Y, Alten R, Caporali R, Durez P, Iannone $\mathrm{F}$, et al. Comparative efficacy of subcutaneous (CT-P13) and intravenous infliximab in adult patients with rheumatoid arthritis: a network metaregression of individual patient data from two randomised trials. Arthritis Res Therapy [Internet]. 2021; 23(1):119. Available from: http://europepmc. org/abstract/MED/33863352. Doi: https://doi.org/ 10.1186/s13075-021-02487-x. https://europepmc. org/articles/PMC8051052. https://europepmc.org/ articles/PMC8051052?pdf=render.

17. Bawazir YM. Clinicodemographic profiles of rheumatoid arthritis patients from a single center in Saudi Arabia. Open Access Rheumatol. 2020;12: 267-75.

18. Yamazaki K, Suzuki E, Ishihara R, Miyamoto T. Obesity and remission rates in Japanese patients with rheumatoid arthritis requiring anti-tumor necrosis factor alpha therapy. Arch Rheumatol. 2020;35(4):600-8.

19. Askling J, Baecklund E, Granath F, Geborek P, Fored M, Backlin C, et al. Anti-tumour necrosis factor therapy in rheumatoid arthritis and risk of malignant lymphomas: relative risks and time trends in the Swedish Biologics Register. Ann Rheum Dis. 2009;68(5):648-53.

20. Gómez-Reino JJ, Carmona L, Valverde VR, Mola EM, Montero MD. Treatment of rheumatoid arthritis with tumor necrosis factor inhibitors may predispose to significant increase in tuberculosis risk: a multicenter active-surveillance report. Arthritis Rheum. 2003;48(8):2122-7.

21. Kremer JM. The CORRONA database. Autoimmun Rev. 2006;5(1):46-54.

22. Richter A, Meißner Y, Strangfeld A, Zink A. Primary and secondary patient data in contrast: the use of observational studies like RABBIT. Clin Exp Rheumatol. 2016;34(5 Suppl 101):S79-s86.

23. Silva-Fernández L, De Cock D, Lunt M, Low AS, Watson KD, Symmons DPM, et al. Serious infection risk after 1 year between patients with rheumatoid arthritis treated with rituximab or with a second TNFi after initial TNFi failure: results from The British Society for Rheumatology Biologics Register for Rheumatoid Arthritis. Rheumatology (Oxford). 2018;57(9):1533-40.

24. Závada J, Uher M, Sisol K, Forejtová Š, Jarošová K, Mann $\mathrm{H}$, et al. A tailored approach to reduce dose of anti-TNF drugs may be equally effective, but substantially less costly than standard dosing in patients with ankylosing spondylitis over 1 year: a propensity score-matched cohort study. Ann Rheum Dis. 2016;75(1):96-102.
25. Santos-Moreno P, Castillo P, Villareal L, Pineda C, Sandoval H, Valencia O. Clinical outcomes of patients with rheumatoid arthritis treated in a disease management program: real-world results. Open Access Rheumatol. 2020;12:249-56.

26. Gudelj Gračanin A, Matić A, Mikula T, Dobša J, Žagar I, Mužinić Marinić L, et al. Influence of biological therapeutics on patient-reported quality-oflife outcomes (Whoqol-Bref), functional scores and disease activity among Croatian patients with rheumatoid arthritis: our experience. Psychiatr Danub. 2020;32(Suppl 4):547-52.

27. Aoki T, Ito H, Ogura T, Hirata A, Nishiwaki Y, Kameda $H$. Association of age with the nonachievement of clinical and functional remission in rheumatoid arthritis. Sci Rep. 2020;10(1):15277.

28. Mease PJ, Stryker S, Liu M, Salim B, Rebello S, Gharaibeh $\mathrm{M}$, et al. Treatment patterns in rheumatoid arthritis patients newly initiated on biologic and conventional synthetic disease-modifying antirheumatic drug therapy and enrolled in a North American clinical registry. Arthritis Res Ther. 2021;23(1):236.

29. Khaliq T, Khan A, Malik IA. Clinical profile and treatment outcomes of patients with rheumatoid arthritis at a tertiary care hospital of Pakistan. J Pak Med Assoc. 2020;70(7):1143-9.

30. Mori S. Management of rheumatoid arthritis patients with interstitial lung disease: safety of biological antirheumatic drugs and assessment of pulmonary fibrosis. Clin Med Insights Circ Respir Pulm Med. 2015;9(Suppl 1):41-9.

31. Novella-Navarro $M$, Plasencia C, Tornero C, Navarro-Compán V, Cabrera-Alarcón JL, PeiteadoLópez D et al. Clinical predictors of multiple failure to biological therapy in patients with rheumatoid arthritis. Arthritis Res Therapy [Internet]. 2020; 22(1):284. Available from: http://europepmc.org/ abstract/MED/33298140. Doi: https://doi.org/10. 1186/s13075-020-02354-1. https://europepmc.org/ articles/PMC7724866. https://europepmc.org/ articles/PMC7724866?pdf=render.

32. Yokota S, Imagawa T, Mori M, Miyamae T, Takei S, Iwata $\mathrm{N}$, et al. Longterm safety and effectiveness of the anti-interleukin 6 receptor monoclonal antibody tocilizumab in patients with systemic juvenile idiopathic arthritis in Japan. J Rheumatol. 2014;41(4):759-67.

33. Pappas DA, Shan Y, Lesperance T, Kricorian G, Karis E, Rebello S, et al. Maintenance of sustained low disease activity or remission in patients with rheumatoid arthritis treated with etanercept monotherapy: results from the Corrona registry. ACR Open Rheumatol. 2020;2(10):588-94. 
34. Hamann P, Holland R, Hyrich K, Pauling JD, Shaddick G, Nightingale A, et al. Factors associated with sustained remission in rheumatoid arthritis in patients treated with anti-tumor necrosis factor. Arthritis Care Res. 2017;69(6):783-93.

35. Greenmyer JR, Stacy JM, Sahmoun AE, Beal JR, Diri E. DAS28-CRP cutoffs for high disease activity and remission are lower than DAS28-ESR in rheumatoid arthritis. ACR Open Rheumatol. 2020;2(9): 507-11.
36. Leong KP, Tan JWL, Gao X, Koh ET. Conversion among the 28-joint count activity indices for rheumatoid arthritis. Eur J Rheumatol. 2020;7(3):105-11.

37. Yun H, Chen L, Xie F, Patel H, Boytsov N, Zhang X, et al. Do patients with moderate or high disease activity escalate rheumatoid arthritis therapy according to treat-to-target principles? Results from the rheumatology informatics system for effectiveness registry of the american college of rheumatology. Arthritis Care Res. 2020;72(2):166-75. 